This is a postprint version of the following published document:

Mateos-Pérez, J. M.; Soto-Montenegro, M. L.; Peña-Zalbidea, S.; Desco, M.; Vaquero, J. J. (2016). "Functional segmentation of dynamic PET studies: Open source implementation and validation of a leader-follower-based algorithm". Computers in Biology and Medicine, v. 69, February, pp. 181-188.

DOI: $10.1016 /$ j.compbiomed.2015.12.012

Proyectos:

PI11/00616

PI14/ 00860

CPII/00005

TEC2014-56600-R

RETIC RD12/0042/0057

RGP0004/2013

(C) Elsevier 2016

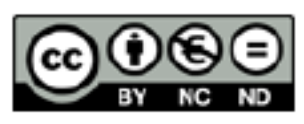

This work is licensed under a Creative Commons Attribution-NonCommercialNoDerivatives 4.0 International License. 


\title{
Functional segmentation of dynamic PET studies: Open source implementation and validation of a leader-follower-based algorithm $\hat{\jmath}^{2}$
}

\author{
Manuel Desco $^{\mathrm{b}, \mathrm{d}}$, Juan José Vaquero ${ }^{\mathrm{b}, \mathrm{d}}$ \\ ${ }^{a}$ Centro de Investigación Biomédica en Red de Salud Mental (CIBERSAM), Madrid, Spain \\ ${ }^{\mathrm{b}}$ Instituto de Investigación Sanitaria Gregorio Marañón, Madrid, Spain \\ ${ }^{\mathrm{c}}$ Montreal Neurological Institute, McGill University, Montreal, Québec, Canada \\ ${ }^{\mathrm{d}}$ Departamento de Bioingeniería e Ingeniería Aeroespacial, Universidad Carlos III de Madrid, Madrid, Spain
}

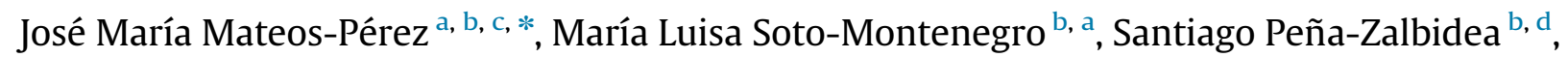

A B S T R A C T

We present a novel segmentation algorithm for dynamic PET studies that groups pixels according to the similarity of their time activity curves. Methods: Sixteen mice bearing a human tumor cell line xenograft (CH 157MN) were imaged with three different ${ }^{68}$ Ga DOTA peptides (DOTANOC, DOTATATE, DOTATOC) using a small animal PET CT scanner. Regional activities (input function and tumor) were obtained after manual delineation of regions of interest over the image. The algorithm was implemented under the jClustering framework and used to extract the same regional activities as in the manual approach. The volume of distribution in the tumor was computed using the Logan linear method. A Kruskal Wallis test was used to investigate significant differences between the manually and automatically obtained volumes of distribution.

Results: The algorithm successfully segmented all the studies. No significant differences were found for the same tracer across different segmentation methods. Manual delineation revealed significant differ ences between DOTANOC and the other two tracers (DOTANOC DOTATATE, $p=0.020$; DOTANOC DOTATOC, $p=0.033$ ). Similar differences were found using the leader follower algorithm.

Conclusion: An open implementation of a novel segmentation method for dynamic PET studies is pre sented and validated in rodent studies. It successfully replicated the manual results obtained in small animal studies, thus making it a reliable substitute for this task and, potentially, for other dynamic segmentation procedures.

Keywords: Dynamic PET; Functional segmentation; Kinetic modeling; Clustering; Open Source

\section{Introduction}

Dynamic nuclear imaging analysis enables quantification of the response of biological tissues more precisely than static analysis in various types of studies, such as those based on myocardial blood flow $[21,9]$ and glucose metabolism [16]. In small animal studies, dynamic imaging based on custom tracers also makes it possible

${ }^{\mathrm{d}}$ Financial support: This work was partially funded by the following grants: Ministerio de Ciencia e Innovación, Instituto de Salud Carlos III (PI11/00616, PI14/ 00860, CPII/00005), Fondo Europeo de Desarrollo Regional (FEDER), "Una manera de hacer Europa"; TEC2014-56600-R, Ministerio de Ciencia e Innovación; RETIC RD12/0042/0057, Ministerio de Economía y Competitividad; and by the Human Frontier Science Program (RGP0004/2013).

* Correspondence to: Montreal Neurological Institute, McGill University, 3801 Rue University, Montréal, Canada QC H3A 2B4. Tel.: +1 514398644.

E-mail address: jmmateos@hggm.es (J.M. Mateos-Pérez). to assess lung inflammation [30] and specific biological receptor activity [15,39].

The quantification process for these studies requires time activity curves (TACs) to be obtained for the relevant organs or tissues, along with the activity generated in arterial blood (known as the input function [IF]). Continuous blood sampling by arterial catheterization is generally considered the gold standard for esti mation of IF [2]. However, the technique is invasive, causes patient discomfort and presents additional difficulties in experiments involving small animals $[27,29,34]$. IF can be obtained directly from image data in human studies under specific conditions, such as the presence of large blood pools in the field of view [43,5]. In small animal studies, it is also possible to extract the image derived IF (IDIF) from the heart or abdominal aorta and use only one or two blood samples to correct for partial volume and spil lover effects [42]. The correct extraction of the IF data using any of 
the previously mentioned techniques is a fundamental step for the adequate quantification of dynamic study results [14,40,44,8].

Typically, extraction of IDIF involves manual delineation of the relevant structures over the image, which is a user dependent and time consuming process [36,41]. During recent years, a number of automatic or semi automatic segmentation approaches have been proposed in the literature $[11,13,17$ 19,22,23,3,6 8]. Several of these methods require a priori knowledge of the number of regions with homogeneous activity into which the image should be partitioned; a classic example is the $k$ means clustering algo rithm [10].

However, in most cases, this knowledge does not exist. In the case of PET, it is common to have several regions whose TACs are not homogeneous owing to contamination from a mixture of the activities of surrounding structures. Therefore, it makes sense to design a clustering approach that divides the image into as many regions with similar kinetic behavior as needed using a similarity metric to establish the threshold for creating a new division. This process has been called functional segmentation (O.G. [32]) to distinguish it from classic segmentation based solely on morpho logical or structural considerations. The methods used for the segmentation of dynamic studies differ from those commonly used to segment static PET images. For an extensive review on these methods see [12].

In this paper, we present an open implementation of a clus tering algorithm based on a leader follower approach that groups voxels with similar TACs. To do so, we aim to replicate the results obtained in [37] by replacing the manual TAC extraction used in that study with the method presented here. We also include a comparison with the classic $k$ means algorithm. Furthermore, we report the results of applying the algorithm using simulated data. Our study extends and validates a preliminary implementation (J. M. [25]).

\section{Materials and methods}

\subsection{Leader follower algorithm: definition and implementation}

Let us consider every dynamic voxel in the image $\boldsymbol{v}=\left\{v_{0}, \ldots\right.$, $\left.\begin{array}{ll}v_{n} & 1\end{array}\right\}$ as a vector consisting of the sequence of different activity levels with time $(t=\{0, \ldots, n 1\})$; this time vector is the TAC for the corresponding voxel. Let us also consider a cluster $\boldsymbol{C}$ as a set of voxels whose centroid $\boldsymbol{c}$ is the average activity for all the voxels in that set.

The classic leader follower formulation uses the following steps [10] to complete the clustering operation:

1. Set a metric and a threshold.

2. Initialize the first cluster with the first voxel in the image.

3. Compute the metric between each voxel and available cluster centroid. If the shortest computed metric is below the thresh old, include that voxel in the corresponding cluster. If not, create a new cluster with that voxel.

4. Repeat until all the voxels have been included in a cluster.

The metric of choice is generally the Euclidean distance; however, since this distance may yield quite different values, it is more convenient for this particular algorithm to use a metric for which a known threshold can be set. For instance, it is possible to use the Pearson or Spearman correlation coefficients and the cosine product, which have values bound in $[1,1]$ and measure the similarity in shape between two given vectors. For this kind of metric, a voxel is included in a given cluster if the similarity measurement is above the threshold. This algorithm is not limited to searching for connected regions: a cluster can include voxels from different spatial locations if they have similar kinetic behavior.

In the particular case of dynamic PET studies, two different tissues or organs may have similar TAC shapes but different activity levels. It is therefore interesting to build clusters with voxels that are not only similar in shape but also in amplitude. For this reason, we modified the classic leader follower algorithm by including additional steps to account for amplitude differences without disregarding shape similarity, as follows:

1. Sort all the voxels in the image by comparing the amplitude. The ones with the greatest maximum amplitude are processed first.

2. Set a similarity metric and a threshold. In this case, the cosine metric is used, which is defined as

$\operatorname{sim}(\boldsymbol{v}, \boldsymbol{c})=\cos \theta=\frac{\boldsymbol{v} \cdot \boldsymbol{c}}{\|\boldsymbol{v}\| \cdot \mid\|\boldsymbol{c}\|}$

where $\boldsymbol{v}$ and $\boldsymbol{c}$ are the voxel TAC vector and the centroid vector for cluster $\mathbf{C}$, respectively. This similarity metric computes the cosine of the angle between two given vectors. Its values are bound in $[1,1]$, which allows the user to set a sensible and meaningful threshold.

3. Initialize the first cluster with the first voxel, following the sorted order.

4. Compute the similarity score between each voxel and the available clusters. If the similarity is above the given threshold, include that cluster in a set of candidates, $\mathbf{C}_{\mathbf{c}}$. If no candidates are found, that voxel is used to create a new cluster.

5. Select a cluster $\mathbf{C}_{i}$ from $\mathbf{C}_{\mathbf{c}}$ such that

$\arg \min D\left(\boldsymbol{v}, \boldsymbol{c}_{i}\right) \cdot e^{-\operatorname{sim}\left(\boldsymbol{v}, \boldsymbol{c}_{i}\right)}$

where $D\left(\boldsymbol{v}, \boldsymbol{c}_{i}\right)$ is the Euclidean distance between the voxel TAC vector $\boldsymbol{v}$ and the centroid from cluster $i$. The exponential term (again using the previously described similarity function) penalizes voxels close in amplitude to the centroid but less similar in shape.

6. Include the voxel in the selected cluster $C_{i}$.

7. Repeat until all the voxels have been assigned.

Step 1 ensures that the next voxel to analyze is always the one with the largest available amplitude. The algorithm is very dependent on the order in which the voxels are processed for cluster creation, because new clusters are created as needed when none of the existing ones have the desired similarity scores. The initial sorting step optimizes the final result by forcing different clusters to include only those voxels with the greatest amplitude available among all those with a similar shape, thus minimizing the influence of noise and spillover effects.

This algorithm was implemented under the jClustering frame work (José María [26]) for the ImageJ platform [35] and has been released under a free software license (GNU GPL); specific imple mentation details can be found in (José María [26]). The source code, along with binary downloads, can be obtained at https:// github.com/HGGM LIM/jclustering/blob/master/src/main/java/ jclustering/techniques/LeaderFollower.java.

\subsection{Experimental protocol}

Sixteen male nude NU/NU mice were used in this study. The animals were purchased from Charles River Laboratories (Spain), maintained at a constant temperature $\left(24 \pm 0.5{ }^{\circ} \mathrm{C}\right)$ under a $12 \mathrm{~h}$ light/dark cycle and permitted free access to commercial rodent laboratory chow and water. All experimental animal procedures were conducted in conformity with European Communities 
Council Directive 2010/63/EU and approved by the Ethics Com mittee for Animal Experimentation of our institution.

$1.5 \cdot 10^{6} \mathrm{CH} 157 \mathrm{MN}$ human tumor cells was injected in both mouse flanks. Imaging was performed 721 days after tumor cell inoculation using a small animal PET CT scanner (ARGUS PET CT, SEDECAL, Madrid). Scans were obtained under anesthesia with isoflurane (3\% induction and $1.5 \%$ maintenance in $100 \% \mathrm{O}_{2}$ ). Three different ${ }^{68} \mathrm{Ga}$ DOTA peptides (referred to in this paper as DOTA NOC, DOTATATE and DOTATOC) were injected into the tail vein (mean activity $11.47 \mathrm{MBq}$, range 9.25 20.35 MBq). Selection of the tracer, day and mouse was randomized. A total of 24 images were acquired, since a mouse could be injected with more than one tracer on more than one day.

\subsection{Image acquisition and manual segmentation}

Tracer injections were followed by a 90 min dynamic PET study. Dynamic data were reformatted into 112 time frames $(20 \mathrm{~s} \times 10 \mathrm{~s}, 10 \mathrm{~s} \times 30 \mathrm{~s}$ and $82 \mathrm{~s} \times 60 \mathrm{~s})$. Frames corresponding to the last 60 minutes of the study were summed to create a static image. Images were reconstructed using a 2D OSEM (Ordered Subset Expectation Maximization) algorithm to provide images with $1.45 \mathrm{~mm}$ full width half maximum (FWHM) resolution (voxel size $0.3875 \times 0.3875 \times 0.7750 \mathrm{~mm}^{3}$ ). The energy window was $400700 \mathrm{keV}$. Decay, dead time and random corrections were applied.

CT images were acquired using the PET/CT scanner described above with standard parameters for tumor imaging: $320 \mathrm{~mA}$, $45 \mathrm{KV}, 360$ projections, 8 shots and $200 \mu \mathrm{m}$ of resolution. CT images were reconstructed using a Feldkamp algorithm to obtain an isotropic voxel size of $0.125 \mathrm{~mm}$ [1]. These anatomical images did not require any registration with their corresponding PET scans thanks to the intrinsic alignment of the PET/CT device.

CT images were used to manually draw the regions of interest (ROI) for the tumors. The IF was obtained by manually delineating the descending aorta in the static PET image. Manual segmenta tions were performed by two operators with more than 5 years' experience and revised by a senior operator with more than 10 years' experience.

\subsection{Automatic functional segmentation process}

\subsubsection{Algorithm presented}

Prior to segmentation, the image volume was masked to eliminate voxels outside the mouse body using a simple thresh olding method.

The proposed leader follower algorithm was applied to the resulting masked volume using different similarity thresholds, as explained in step 2 of the algorithm workflow (selected values are $0.4,0.5,0.6,0.7$ and 0.8 ). The centroids for the selected clusters were the TACs to be used as input for the Logan analysis. A suc cessful segmentation is defined as that which enables clear dif ferentiation of at least the IF and the tumor tissue from the sur rounding organs. Clusters were selected by visual identification.

\subsection{2. $k$ means}

For comparison purposes, the classical $k$ means algorithm was also used to segment the studies and obtain the TACs for the relevant tissues. $k$ means was selected due to its status as a baseline algorithm and for its availability in a wide range of soft ware libraries. For the comparison to be as uniform as possible, the fixed number of clusters was chosen according to the average number of clusters generated by the leader follower method across all studies when the threshold was set at 0.6 .

\subsection{Quantification}

In order to contrast both methods (automatic and manual), we directly compared the post processed volumes of distribution after the kinetic analysis and not the TACs obtained, because TACs that are apparently similar may yield very different volume of distribution values. The volume of distribution was obtained for both the manual and the computer assisted segmentations using the Logan plot [20]. The model was resolved using the traceRki netic modeling library ([24]). $t^{*}$ time was automatically chosen so that a $<10 \%$ error was obtained for the linear regression. This criterion is the same as that applied in other kinetic analysis tools, such as PMOD [4].

\subsection{Statistical analysis}

Paired Wilcoxon rank sum tests were used to determine whe ther the segmentations provided by the algorithm yielded results that were significantly different from the manual ones within the same tracer. No multiple comparison correction was used for these tests.

The differences in the volume of distribution for the different tracers within the same segmentation method, including manual delineation, were also tested. Differences between tracers were compared using a Kruskal Wallis test, which was followed by a pairwise Wilcoxon rank test with the Bonferroni correction for multiple comparisons. Results were considered significant for $p<0.05$.

The statistical analysis was performed using the statistical package R v3.0.0 (R [31]).

\subsection{Simulated data}

The validation procedure based on simulated data can be found in Supplementary material File 1.

\section{Results}

Fig. 1 shows several static frames from the acquired data. The algorithm presented was successful in separating the desired regions when the threshold values were $0.6,0.7$ or 0.8 (Fig. 2). Segmentation was not successful in some of the studies when the lowest threshold values were used ( 0.4 and 0.5$)$, and no statistical results are reported here for these values. Fig. 3 shows examples of the TACs obtained using the algorithm along with the manual results.

The total computation time taken by each algorithm and the final number of clusters created depending on the threshold are shown in Table 1.

The results for the statistical analysis are summarized in Fig. 4 and Table 2. No significant differences in volume of distribution were found within the same tracer between the manual method and the computer assisted method. The statistical analysis revealed significant differences (Kruskal Wallis p value $=0.0108$ ) between DOTANOC and DOTATATE $(\mathrm{p}=0.020)$ and DOTANOC and DOTATOC $(\mathrm{p}=0.033)$ for the manual segmentation method; simi lar differences were found for all values obtained with the algo rithm for which statistical results are reported (Kruskal Wallis $\mathrm{p}$ values for each threshold $\{0.6,0.7$ and 0.8$\}=0.0019,0.0023$ and 0.0013 ; see Fig. 4 for pairwise p values).

The segmentation with $k$ means $(k=150)$ yielded higher volume of distribution values than those obtained either manually or using the algorithm $($ DOTANOC $=0.873 \pm 0.09$; DOTATATE $=0.975 \pm 0.07$; DOTATOC $=0.987 \pm 0.11$ ). All these values were different to the manual ones $(p<0.05)$; furthermore, the difference between the 

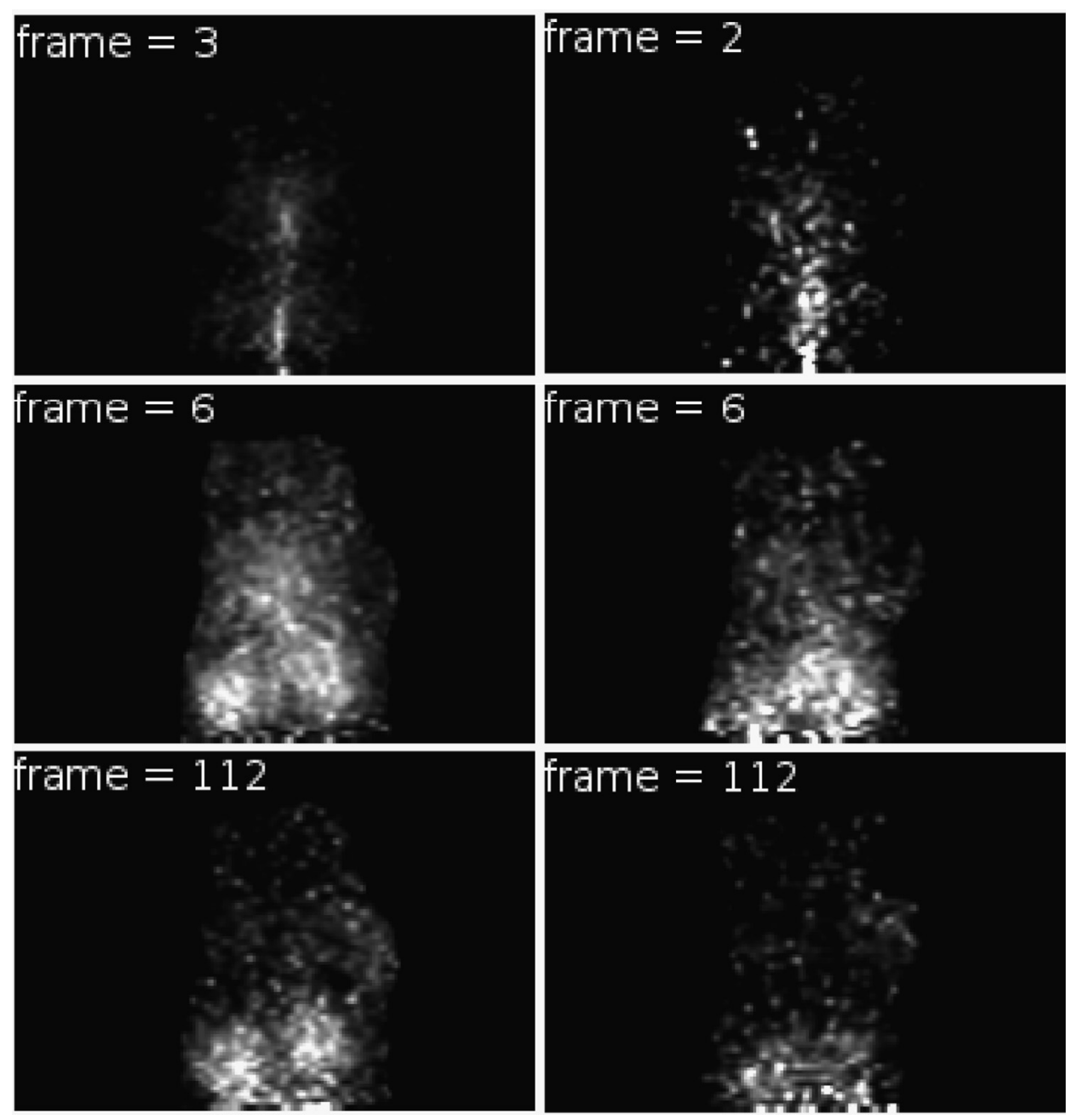

Fig. 1. Three frames from two different mice (left and right) acquired for this study.

tracers DOTANOC and DOTATOC, found manually and with the leader follower algorithm, was not statistically significant. More results from the $k$ mean segmentation can be found in Supple mentary Material File 2.

\section{Discussion}

In this paper, we present a novel segmentation algorithm based on a leader follower approach that, instead of setting a final number of clusters, creates as many clusters as needed depending on a similarity threshold set by the user. In our tests, the algorithm successfully extracted the TACs for the relevant tissues or regions when the threshold was above a certain critical value, and the results were comparable with those obtained in [37]. Images with lower injected radioactivity, which tend to be more affected by noise, were separated into more clusters for the same threshold value. This approach ensures the basic assumption of kinetic analysis, namely, homogeneous kinetic behavior of the voxels assigned to a particular region [28,38]. The algorithm has been implemented using an open clustering framework (José María [26]) and is also available under an open source license (GNU GPL).

Our algorithm addresses one of the weaknesses of the $k$ means algorithm, in which the user needs to run the algorithm several times before reaching an optimal solution because of the random initialization step [45]. From this perspective, our algorithm ensures the reproducibility of the segmentation results and provides a result that does not change for each run. It has to be noted that the result might not be the optimal one, even in the manual case. Our rationale is that the comparison against manual segmentation helps to test the algorithm in a real world envir onment. This validation approach shows that the algorithm is a viable substitute for manual tasks. This finding is also supported by the results of applying this algorithm to a set of simulated data (see Supplementary material File 1), which shows excellent results in recovering the original TACs at least as well as manual segmentation.

We showed that the algorithm was stable with respect to the TACs obtained after applying the algorithm, we confirmed that the algorithm is stable: the variation of the threshold value does not cause significant changes in the volume of distribution for each tracer (Fig. 5), since the differences between the tracers and the sign of those differences are maintained. As this threshold value is increased, the variability of the volume of distribution decreases. We hypothesize that this is due to the reduction in the region size obtained when the similarity threshold is increased (Fig. 2(b)). Therefore, TAC heterogeneity decreases owing to partial volume effects or noise, as the area included in the cluster is concentrated on the center of the region, where voxels are less mixed with activity from surrounding organs and tissues [23]. This effect is seen clearly in Fig. 2(c), where the tumor region is surrounded by smaller clusters that are heavily affected by spillover. Those small clusters are not included in the segmented tissue because their TACs do not conform to the similarity or distance constraints 
a
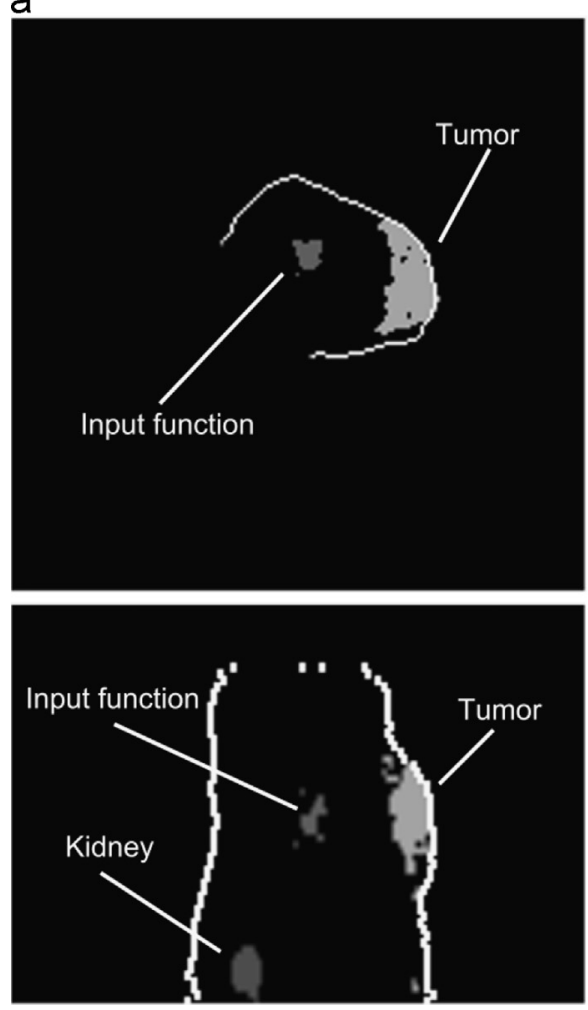
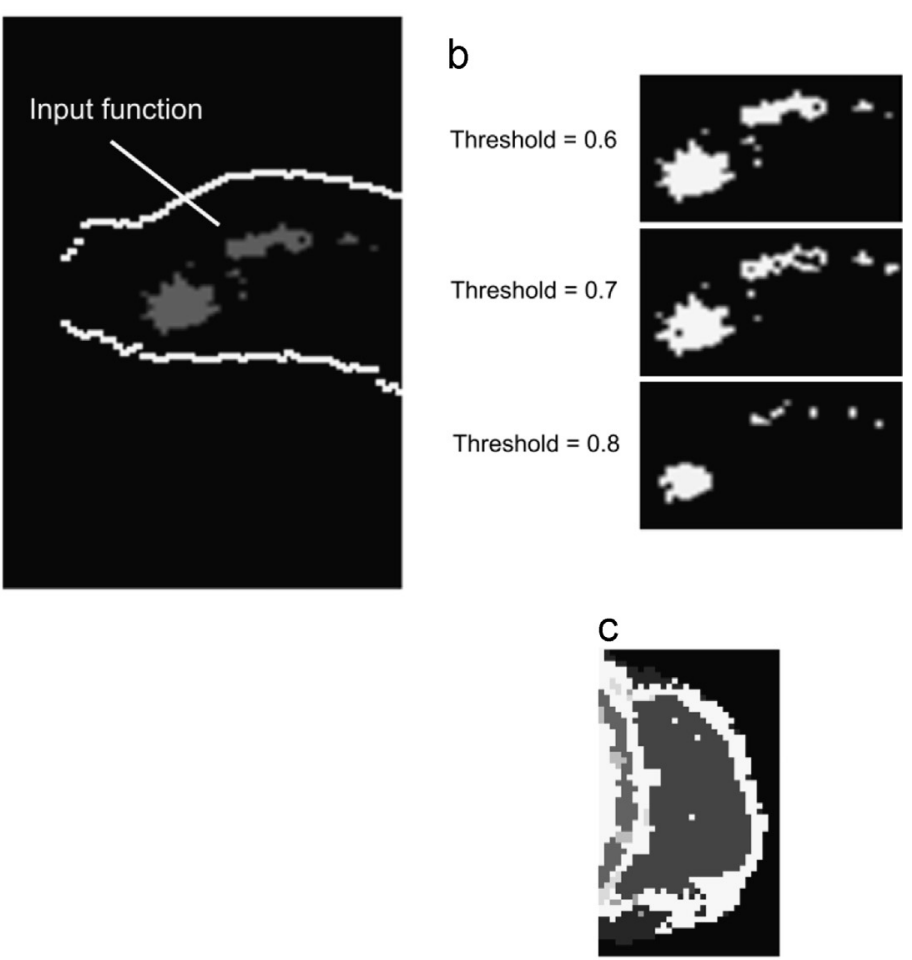

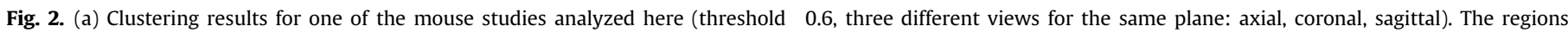

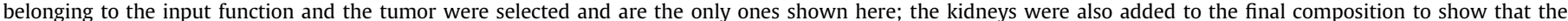

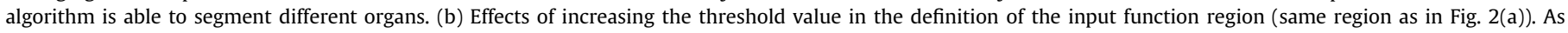

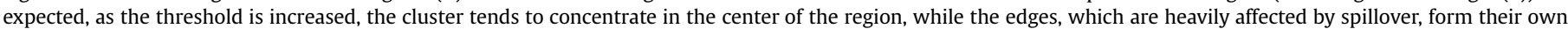

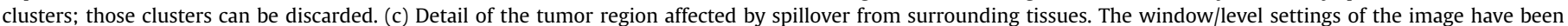
adjusted to clearly visualize the tumor region; surrounding clusters are shown in white, although they do not necessarily form a single connected region.
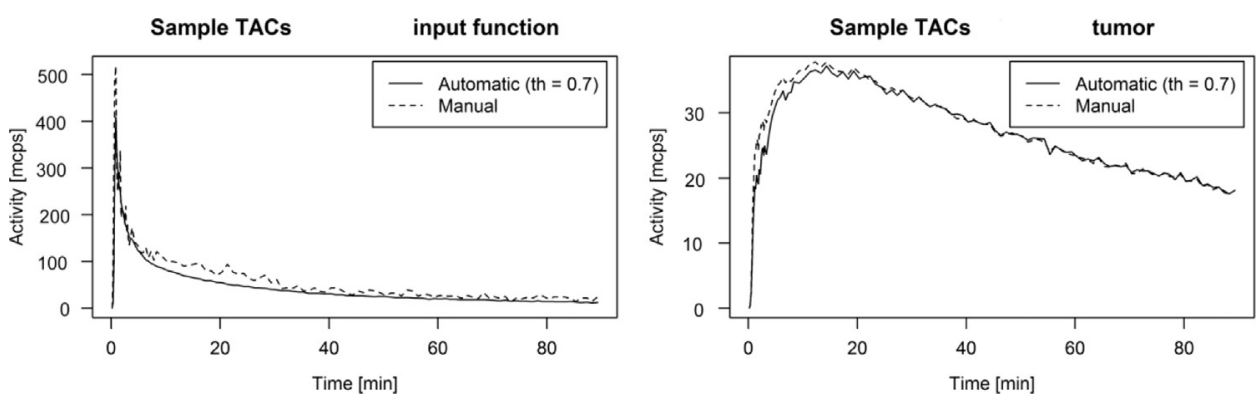

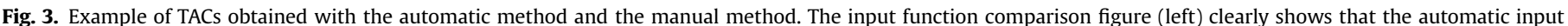
function presents smaller noise levels than the manually obtained one. Tumor TACs remain very similar, with virtually no differences between them.

Table 1

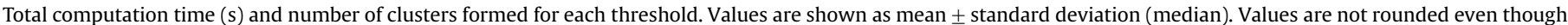

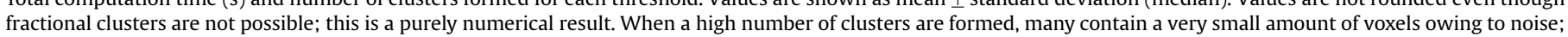
given that the user interface presents the clusters by size (largest first), the relevant ones will always be among the first to be inspected by the user.

\begin{tabular}{lcrr}
\hline & Threshold 0.6 & Threshold 0.7 & Threshold 0.8 \\
\hline Time $(s)$ & $18.1 \pm 4.8(17.8)$ & $26.3 \pm 18.2(22.2)$ & $67.6 \pm 92.9(34.61)$ \\
\# of clusters & $149.5 \pm 143.9(102.5)$ & $611.2 \pm 820.8(297)$ & $2255.9 \pm 2476.1(1375.5)$ \\
\hline
\end{tabular}

imposed by the algorithm. This same effect also applies to TACs heavily affected by noise: in low activity studies, noise can distort the activity signal to the point of making the algorithm create a new cluster for the regions with the lowest activities.

While the manually segmented regions and the ones obtained using the algorithm tended to overlap (Fig. 5), they are not iden tical. Some of the segmented tumors show internal heterogeneity, that is, they are often divided into high uptake and low uptake regions, probably because of necrosis. As the tumor ROIs were drawn manually over the CT image, where they appear homo geneous, the functional differences observed were not taken into account, and in some cases, necrotic tissue, which is usually pre sent in the center of the tumor region, was included. This higher sensitivity of the algorithm may account for the slightly higher overall volume of distribution (Table 2), as the tumor TAC was extracted using only the high uptake areas. This manual 
segmentation error is shared among the different tracers, and its overall effect can be disregarded if the final objective is to evaluate which tracer has the greatest uptake in the tumor tissue. However, it is important to consider when the quantification measurements need a precise result that accounts faithfully for the volume of distribution in the active tumor; in this respect, the same regions of interest could have been drawn using the summed PET image. Furthermore, it is common practice to obtain the IF from a very small region to minimize the influence of spillover. In that respect, when the segmentation process aims to extract the TAC for each tissue, region overlap is not as useful for purposes of comparison as the results of the kinetic analysis.

The comparison against $k$ means showed that the present algorithm was more precise when separating the different regions, even though the number of clusters chosen for this comparison was reasonably high (150). Several factors may account for these changes, although we hypothesize that this might be due to the region growing approach (starting from high amplitude seed points) used by the algorithm, which is better suited for this particular application. In any case, the region growing approach proves that the leader follower modification proposed is not simply an inverse $k$ means algorithm that substitutes a fixed number of clusters with a fixed similarity threshold.

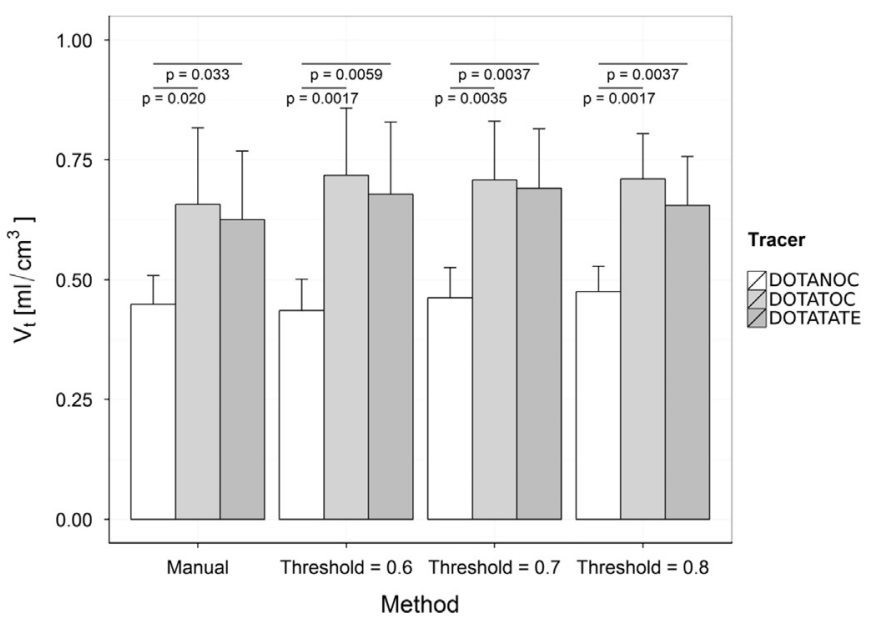

Fig. 4. Volumes of distribution for the various segmentation procedures (manual and automatic with different thresholds). $p$ values within the same procedure are adjusted for multiple comparisons using the Bonferroni correction.

Table 2

Volume of distribution values per tracer and segmentation method; p values are greater than the significance level $(0.05)$ for the observed differences. $V_{t}$ values are shown as mean \pm standard deviation.

\begin{tabular}{lllll}
\hline & Manual & Threshold 0.6 & Threshold 0.7 & Threshold 0.8 \\
\hline DOTANOC & $0.45 \pm 0.06$ & $0.44 \pm 0.07$ & $0.46 \pm 0.06$ & $0.47 \pm 0.05$ \\
DOTATATE & $0.66 \pm 0.16$ & $0.72 \pm 0.14$ & $0.71 \pm 0.12$ & $0.71 \pm 0.09$ \\
DOTATOC & $0.63 \pm 0.14$ & $0.68 \pm 0.15$ & $0.69 \pm 0.12$ & $0.65 \pm 0.10$ \\
\hline
\end{tabular}

Our study has a number of limitations. The algorithm does not include any partial volume or spillover correction logic, such as the geometric transfer matrix algorithm (Olivier G [33]); however, this is not the primary purpose of the algorithm, which is intended to replicate the results obtained from manual segmentation. Since our small animal studies did not use blood sampling, the volume of distribution values are potentially biased. However, the valida tion process has been used to show that both the manual and the computer assisted segmentation approaches yield TACs that lead to equivalent results. In addition, although for this particular application we found no differences in the threshold values used to segment the image, a successful segmentation may not have held true for other applications or for other quantification analyses.

The similarity threshold may take arbitrary values and, even if its mathematical significance is clear, the practical result is subject to a process of trial and error, as the final number of clusters depends on several factors, including image noise. In any case, as the algorithm is fast, different values can be tested until the relevant structures can be discerned visually. Table 2 shows the computation times and the number of clusters created. A high threshold value (i.e. 0.8 ) over segments the data, but most of the clusters created are only a few pixels in size and can be ruled out as noise. Furthermore, as the algorithm presents the results so that larger clusters (i.e., those including more voxels) are shown first, it is easy to locate the relevant organs in the user interface. Since higher values also cause the final result to stay closer to the center of the region (Fig. 2(b)), a balance needs to be achieved: larger threshold values imply more concentrated clusters, but also more fragmentation.

Finally, although we validated the algorithm using the cosine similarity metric, other similarity metrics, such as the Pearson correlation score or the Spearman rank correlation coefficient, have also been implemented and yielded similar results in pre liminary tests, albeit with higher computation times.

\section{Conclusion}

We present an open implementation of a segmentation algo rithm for dynamic PET studies based on grouping voxels with similar TACs. The algorithm can be used to successfully replicate results obtained from manual segmentations performed in small animal studies using ${ }^{68} \mathrm{Ga}$ DOTA peptides, thus making it a reli able substitute for this task and, potentially, for other dynamic segmentation procedures. This leader follower based algorithm is fast, has a publicly available source code and can be used directly from the Image image analysis and processing platform.

\section{Conflict of interest statement}

The authors declare that they have no conflicts of interest.
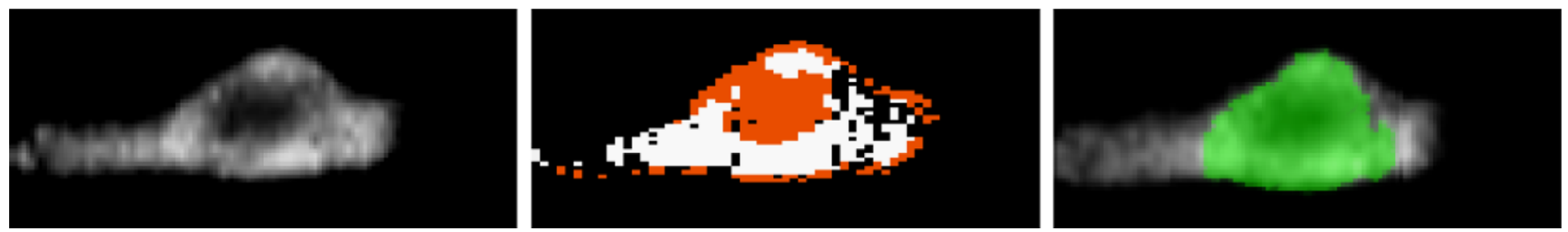

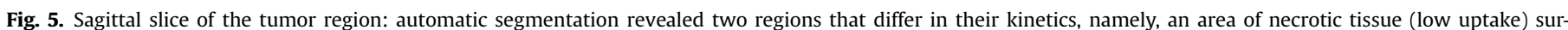

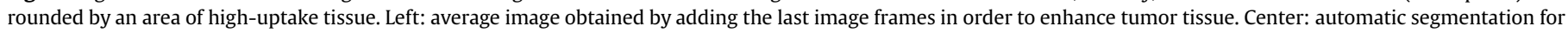

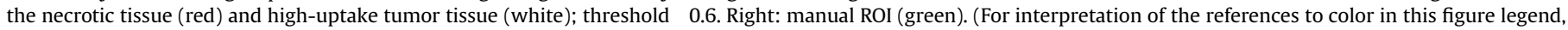
the reader is referred to the web version of this article.) 


\section{Acknowledgments}

The authors thank Alexandra de Francisco and Yolanda Sierra for their invaluable help during animal handling and image acquisition and Steve Bacharach for his excellent advice and con tributions. Special thanks are due to Ángel Udías and Elena Her ranz for providing simulated data. The authors also wish to thank the editorial board of this journal for their support during the review of the manuscript.

\section{Appendix A. Supplementary material}

Supplementary data associated with this article can be found in the online version at http://dx.doi.org/10.1016/j.compbiomed.2015. 12.012 .

\section{References}

[1] M. Abella, J.J. Vaquero, A. Sisniega, J. Pascau, A. Udías, V. García, I. Vidal, M. Desco, Software Architecture for Multi-Bed FDK-Based Reconstruction in XRay CT Scanners, Comput. Methods Prog. Biomed.. 107 (2012) 218-232, http: //dx.doi.org/10.1016/j.cmpb.2011.06.008.

[2] M. Bentourkia, A. Bol, A. Ivanoiu, C. Michel, A. Coppens, M. Sibomana, G. Cosnard, A.G. De Volder, A Standardized Blood Sampling Scheme in Quantitative FDG-PET Studies, IEEE Trans. Med. Imaging 18 (5) (1999) 379-384, http://dx.doi.org/10.1109/42.774165.

[3] J.G. Brankov, N.P. Galatsanos, M.N. Wernick, Segmentation of Dynamic PET or fMRI Images Based on a Similarity Metric, IEEE Trans. Nucl. Sci. 50 (5) (2003) 1410-1414, http://dx.doi.org/10.1109/TNS.2003.817963.

[4] Cyrill Burger, Alfred Buck, Requirements and implementation of a flexible kinetic modeling tool, J. Nucl. Med.: Off. Publ. Soc. Nucl. Med. 38 (11) (1997) 1818-1823 〈http://www.ncbi.nlm.nih.gov/pubmed/9374364〉.

[5] Kewei Chen, Daniel Bandy, Eric Reiman, Sung-Cheng Huang, Michael Lawson, Dagan Feng, Lang-sheng Yun, Anita Palant, Noninvasive quantification of the Cerebral Metabolic Rate for Glucose Using Positron Emission Tomography, 18FFluoro-2-Deoxyglucose, the Patlak Method, and an Image-Derived Input Function, J. Cereb. Blood Flow Metab. 18 (7) (1998) 716-723, http://dx.doi.org/ 10.1097/00004647-199807000-00002.

[6] Jinxiu Cheng-Liao, Jinyi Qi, Segmentation of Mouse Dynamic PET Images Using a Multiphase Level Set Method, Phys. Med. Biol. 55 (21) (2010) 6549-6569, http://dx.doi.org/10.1088/0031-9155/55/21/014.

[7] S.K. Choy, M.L. Tang, C.Z. Tong, Image segmentation using fuzzy region competition and spatial/frequency information, IEEE Trans. Image Process. 20 (99) (2011) 1473-1484 , IEEE/http://ieeexplore.ieee.org/xpls/abs_all.jsp? arnumber 5643926)

[8] Etienne Croteau, Eric Lavallée, Sébastien M. Labbe, Laurent Hubert, Fabien Pifferi, Jacques A. Rousseau, Stephen C. Cunnane, André C. Carpentier, Roger Lecomte, François Bénard, Image-derived input function in dynamic human PET/CT: methodology and validation with 11C-Acetate and 18FFluorothioheptadecanoic acid in muscle and 18F-Fluorodeoxyglucose in brain, Eur. J. Nucl. Med. Mol. Imaging, 37, (2010) 1539-1550, http://dx.doi.org/ 10.1007/s00259-010-1443-z.

[9] Robert A. Dekemp, Jerome Declerck, Ran Klein, Xiao-Bo Pan, Ryo Nakazato, Christine Tonge, Parthiban Arumugam, et al., Multisoftware reproducibility study of stress and rest myocardial blood flow assessed with 3D Dynamic PET/ $\mathrm{CT}$ and a 1-Tissue-Compartment Model of 82Rb Kinetics, J. Nucl. Med.: Off. Publ. Soc. Nucl. Med. 54 (2013) 571-577, http://dx.doi.org/10.2967/ jnumed.112.112219.

[10] Richard O. Duda, Peter E. Hart, David G. Stork, Pattern Classification, WileyInterscience, New York, 2001.

[11] Georges El Fakhri, Arkadiusz Sitek, Bastien Guérin, Marie Foley Kijewski, Marcelo F. Di Carli, Stephen C. Moore, Quantitative dynamic cardiac 82Rb PET using generalized factor and compartment analyses, J. Nucl. Med.: Off. Publ. Soc. Nucl. Med. 46 (8) (2005) 1264-1271 〈http://www.ncbi.nlm.nih.gov/ pubmed/16085581>.

[12] Brent Foster, Ulas Bagci, Awais Mansoor, Ziyue Xu, Daniel J. Mollura, A Review on Segmentation of Positron Emission Tomography Images, Comput. Biol. Med. 50 (2014) 76-96, http://dx.doi.org/10.1016/j.compbiomed.2014.04.014.

[13] Daniel Gutierrez, Marie-Louise Montandon, Frédéric Assal, Mohamed Allaoua, Osman Ratib, Karl-Olof Lövblad, Habib Zaidi, Anatomically guided voxel-based partial volume effect correction in brain PET: impact of MRI segmentation, Comput. Med. Imaging Graph.: Off. J. Comput. Med. Imaging Soc. 36 (8) (2012) 610-619, http://dx.doi.org/10.1016/j.compmedimag.2012.09.001.

[14] Sara L. Hackett, Dan Liu, Anastasia Chalkidou, Paul Marsden, David Landau, John D. Fenwick, Estimation of Input Functions from Dynamic [18F]FLT PET Studies of the Head and Neck with Correction for Partial Volume Effects, EJNMMI Res. 3 (1) (2013) 84, http://dx.doi.org/10.1186/2191-219X-3-84.
[15] Eric D. Hostetler, Sandra Sanabria-Bohórquez, Waisi Eng, Aniket D. Joshi, Shailendra Patel, Raymond E. Gibson, Stacey O'Malley, et al., Evaluation of [(18)F]MK-0911, a Positron Emission Tomography (PET) Tracer for Opioid Receptor-Like 1 (ORL1), in Rhesus Monkey and Human, Neurolmage 68 (2012) 1-10, http://dx.doi.org/10.1016/j.neuroimage.2012.11.053.

[16] Marc C. Huisman, Larissa W. van Golen, Nikie J. Hoetjes, Henri N. Greuter, Patrick Schober, Richard G. Ijzerman, Michaela Diamant, Adriaan A. Lammertsma, Cerebral blood flow and glucose metabolism in healthy volunteers measured using a high-resolution PET scanner, EJNMMI Res. 2 (1) (2012) 63, http://dx.doi.org/10.1186/2191-219X-2-63.

[17] Chietsugu Katoh, Keiichiro Yoshinaga, Ran Klein, Katsuhiko Kasai, Yuuki Tomiyama, Osamu Manabe, Masanao Naya, et al., Quantification of regional myocardial blood flow estimation with three-dimensional dynamic rubidium-82 PET and modified spillover correction model, J. Nucl. Cardiol.: Off. Publ. Am. Soc. Nucl. Cardiol. 19 (2012) 763-764, http://dx.doi.org/10.1007/ s12350-012-9558-1.

[18] Jhih-Shian Lee, Kuan-Hao Su, Wen-Yuan Chang, Jyh-Cheng Chen, Extraction of an input function from dynamic micro-PET images using wavelet packet based sub-band decomposition independent component analysis, NeuroImage 63 (2012) 1273-1284 〈http://www.ncbi.nlm.nih.gov/pubmed/22892332〉.

[19] Matthew Liptrot, Karen H. Adams, Lars Martiny, Lars H. Pinborg, Markus N. Lonsdale, Niels V. Olsen, S.øren Holm, Claus Svarer, Gitte M. Knudsen, Cluster analysis in kinetic modelling of the brain: a noninvasive alternative to arterial sampling, Neurolmage 21 (2) (2004) 483-493<http://www.science direct.com/science/article/B6WNP-4BWTPJ5-1/2/ de99c9bbe37cda3bfa08ed714fe9ad7b $\rangle$.

[20] J. Logan, J.S. Fowler, N.D. Volkow, A.P. Wolf, S.L. Dewey, D.J. Schlyer, R. R. MacGregor, R. Hitzemann, B. Bendriem, S.J. Gatley, Graphical analysis of reversible radioligand binding from time-activity measurements applied to [N-11C-Methyl]-(-)-Cocaine PET studies in human subjects, J. Cereb. Blood Flow. Metab.: Off. J. Int. Soc. Cereb. Blood Flow Metab. 10 (5) (1990) 740-747, http://dx.doi.org/10.1038/jcbfm.1990.127.

[21] Mireille Lortie, Rob S.B. Beanlands, Keiichiro Yoshinaga, Ran Klein, Jean N. Dasilva, Robert A. DeKemp, Quantification of myocardial blood flow with 82Rb dynamic PET imaging, Eur. J. Nucl. Med. Mol. Imaging 34 (11) (2007) 1765-1774, http://dx.doi.org/10.1007/s00259-007-0478-2.

[22] R. Mabrouk, F. Dubeau, L. Bentabet, Dynamic cardiac PET imaging : extraction of time-activity curves using ICA and a generalized gaussian distribution model, IEEE Trans. Biomed. Eng. 60 (2012) 63-71, http://dx.doi.org/10.1109/ TBME.2012.2221463 (PP (99).

[23] Renaud Maroy, Raphaël Boisgard, Claude Comtat, Vincent Frouin, Pascal Cathier, Edouard Duchesnay, Frédéric Dollé, Peter E. Nielsen, R. égine Trébossen, Bertrand Tavitian, Segmentation of Rodent Whole-Body Dynamic PET Images: an Unsupervised Method Based on Voxel Dynamics, IEEE Trans. Med. Imaging 27 (3) (2008) 342-354, http://dx.doi.org/10.1109/ TMI.2007.905106.

[24] J.M. Mateos-Pérez, M. Desco, J.J. Vaquero. Tracer Kinetic Modeling with R for Batch Processing of Dynamic PET Studies, in: edited by Laura M Roa Romero, Proceedings of the XIII Mediterranean Conference on Medical and Biological Engineering and Computing 2013 SE-75, 41:301-4. IFMBE Proceedings. Springer International Publishing, 2014, doi:10.1007/978-3-319-00846-2_75.

[25] J.M., Mateos-Pérez, J.J. Vaquero, C. García-Villalba, L. Cusso, M. Desco. Leaderfollower clustering algorithm for automatic segmentation of cardiac PET studies, in: Proceedings of 2011 IEEE Nuclear Science Symposium Conference Record, 2011, 3133-36. IEEE. doi:10.1109/NSSMIC.2011.6152570.

[26] José María Mateos-Pérez, Carmen García-Villalba, Javier Pascau, Manuel Desco, Juan J. Vaquero, jClustering, an open framework for the development of 4D clustering algorithms (Edited by Lennart Martens), PLoS ONE 8 (8) (2013) e70797, http://dx.doi.org/10.1371/journal.pone.0070797.

[27] Philipp T. Meyer, Valentina Circiumaru, Christopher A. Cardi, Daniel H. Thomas, Harshali Bal, Paul D. Acton, Simplified Quantification of Small Animal [18F]FDG PET Studies Using a Standard Arterial Input Function, Eur. J. Nucl. Med. Mol. Imaging 33 (8) (2006) 948-954, http://dx.doi.org/10.1007/ s00259-006-0121-7.

[28] Evan D. Morris, Christopher J. Endres, Kathleen C. Schmid, Bradley T. Christian, Raymond F. Muzic Jr., Ronald E., Fisher. Kinetic modeling in positron emission tomography, In: Miles N. Wernick, John N. Aarsvold, (Eds.), Emission Tomography: The Fundamentals of PET and SPECT, Elsevier, Amsterdam, pp. 499540.

[29] Frédéric Pain, Philippe Lanièce, Roland Mastrippolito, Philippe Gervais, Philippe Hantraye, Laurent Besret, Arterial Input Function Measurement without Blood Sampling Using a Beta-Microprobe in Rats, J. Nucl. Med.: Off. Publ., Soc. Nucl. Med. 45 (9) (2004) 1577-1582〈http://www.ncbi.nlm.nih.gov/ pubmed/15347727>.

[30] Carlos Pérez-Campaña, Vanessa Gómez-Vallejo, Maria Puigivila, Abraham Martin, Teresa Calvo-Fernández, Sergio E. Moya, Søren Thor Larsen, Juan D. Gispert, Jordi Llop, Assessing Lung inflammation after nanoparticle inhalation using 2-Deoxy-2-[(18)F]fluoro-D-Glucose positron emission tomography imaging, Mol. Imaging Biol.: MIB : Off. Publ. Acad. Mol. Imaging 16 (2) (2013) 264-273, http://dx.doi.org/10.1007/s11307-013-0682-3.

[31] R. Core Team, R: A Language and Environment for Statistical Computing, GBIF, Vienna, Austria, 2013 〈http://www.r-project.org .

[32] O.G. Rousset, Yilong Ma, A.C. Evans, Correction for partial volume effects in PET: principle and validation, J. Nucl. Med. 39 (5) (1998) 904 (Soc Nuclear Med)〈http://jnm.snmjournals.org/cgi/content/abstract/39/5/904)〉. 
[33] Olivier G. Rousset, D. Louis Collins, Arman Rahmim, Dean F. Wong, Design and Implementation of an automated partial volume correction in PET: application to dopamine receptor quantification in the normal human striatum, J. Nucl. Med.: Off. Publ., Soc. Nucl. Med. 49 (7) (2008) 1097-1106, http://dx.doi.org/ 10.2967/jnumed.107.048330.

[34] Martin Schain, Simon Benjaminsson, Katarina Varnäs, Anton Forsberg Christer Halldin, Anders Lansner, Lars Farde, Andrea Varrone, Arterial input function derived from pairwise correlations between PET-Image voxels, J. Cereb. Blood Flow. Metab.: Off. J. Int. Soc. Cereb. Blood Flow Metab. 33 (7) (2013) 1058-1065, http://dx.doi.org/10.1038/jcbfm.2013.47.

[35] Caroline A. Schneider, Wayne S. Rasband, Kevin W. Eliceiri, NIH Image to ImageJ: 25 years of image analysis, Nat. Methods 9 (7) (2012) 671-675, http: //dx.doi.org/10.1038/nmeth.2089.

[36] Piotr J. Slomka, Erick Alexanderson, Rodrigo Jácome, Moises Jiménez, Edgar Romero, Aloha Meave, Ludovic Le Meunier, et al., Comparison of clinical tools for measurements of regional stress and rest myocardial blood flow assessed with 13N-ammonia PET/CT, J. Nucl. Med.: Off. Publ., Soc. Nucl. Med. 53 (2) (2012) 171-181, http://dx.doi.org/10.2967/jnumed.111.095398.

[37] María Luisa Soto-Montenegro, Peña-Zalbidea Santiago, Jose María MateosPérez, Marta Oteo, Eduardo Romero, Miguel Ángel Morcillo, Manuel Desco, Meningiomas: A Comparative Study of 68Ga-DOTATOC, 68Ga-DOTANOC and 68Ga-DOTATATE for Molecular Imaging in Mice, PLoS ONE 9 (11) (2014) e111624, http://dx.doi.org/10.1371/journal.pone.0111624.

[38] Mustafa Takesh, The potential benefit by application of kinetic analysis of PET in the clinical oncology, ISRN Oncol. 2012 (2012) 349351, http://dx.doi.org/ $10.5402 / 2012 / 349351$.

[39] Tetsuya Tsujikawa, Sami S. Zoghbi, Jinsoo Hong, Sean R. Donohue, Kimberly J. Jenko, Robert L. Gladding, Christer Halldin, Victor W. Pike, Robert B. Innis, Masahiro Fujita, In Vitro and in Vivo Evaluation of (11)C-SD5024, a Novel PET radioligand for human brain imaging of cannabinoid CB1 receptors, NeuroImage, 84, (2013) 1-9, http://dx.doi.org/10.1016/j.neuroimage.2013.09.043.
[40] I.N. Weinberg, S.C. Huang, E.J. Hoffman, L. Araujo, C. Nienaber, M. Grover McKay, M. Dahlbom, $\mathrm{H}$. Schelbert, Validation of PET-Acquired Input Function for Cardiac Studies, J. Nucl. Med. 29 (2) (1988) 241-247〈http://jnm.snmjour nals.org/cgi/content/abstract/29/2/241>.

[41] K.P. Wong, D. Feng, S.R. Meikle, M.J. Fulham, Segmentation of dynamic PET images using cluster analysis, IEEE Trans. Nucl. Sci. 49 (1) (2002) 200-207, http://dx.doi.org/10.1109/TNS.2002.998752.

[42] Guoming Xiong, Cumming Paul, Andrei Todica, Marcus Hacker, Peter Bartenstein, Guido Böning, Noninvasive image derived heart input function for CMRglc measurements in small animal slow infusion FDG PET studies, Phys. Med. Biol. 57 (23) (2012) 8041-8059, http://dx.doi.org/10.1088/ 0031-9155/57/23/8041.

[43] Paolo Zanotti-Fregonara, Kewei Chen, Jeih-San Liow, Masahiro Fujita, Robert B. Innis, Image-Derived input function for brain PET studies: many challenges and few opportunities, J. Cereb. Blood Flow. Metab.: Off. J. Int. Soc. Cereb. Blood Flow. Metab. 31 (10) (2011) 1986-1998, http://dx.doi.org/10.1038/ jcbfm.2011.107.

[44] Paolo Zanotti-Fregonara, El. Mostafa Fadaili, Renaud Maroy, Claude Comtat, Antoine Souloumiac, Sebastien Jan, Maria-Joao Ribeiro, V.éronique Gaura, Avner Bar-Hen, R.égine Trébossen, Comparison of eight methods for the estimation of the image-derived input function in dynamic [(18)F]-FDG PET human brain studies, J. Cereb. Blood Flow. Metab.: Off. J. Int. Soc. Cereb. Blood Flow. Metab. 29 (11) (2009) 1825-1835, http://dx.doi.org/10.1038/ jcbfm.2009.93.

[45] Paolo Zanotti-Fregonara, Renaud Maroy, Claude Comtat, Sebastien Jan, V. éronique Gaura, Avner Bar-Hen, Maria-Joao Ribeiro, R.égine Trébossen, Comparison of 3 methods of automated internal carotid segmentation in human brain PET Studies: application to the estimation of arterial input function, J. Nucl. Med.: Off. Publ., Soc. Nucl. Med. 50 (3) (2009) 461-467, http://dx.doi org/10.2967/jnumed.108.059642. 